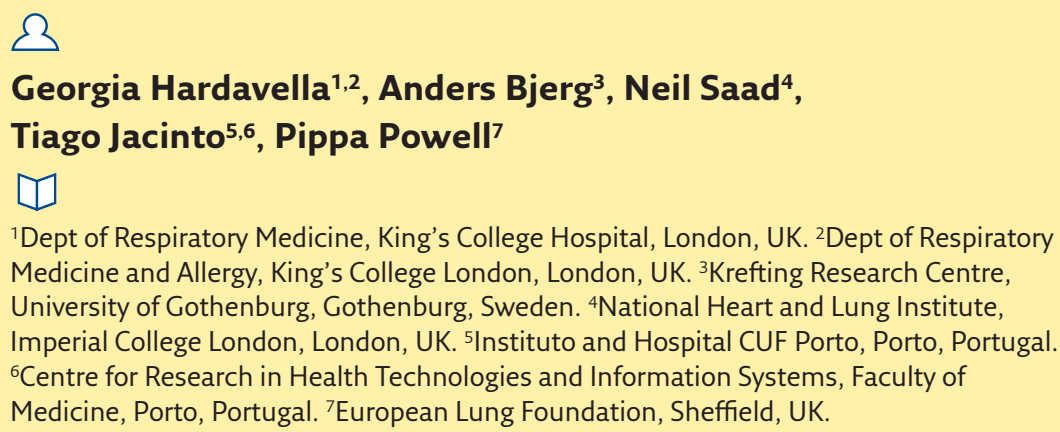

\title{
How to optimise patient and public involvement in your research
}

\section{Doing science}

Over the past few years, patient and public involvement (PPI) in research has often been a key component of successful clinical research grant applications. However, junior researchers and many supervisors often seem rather uncomfortable when filling in this part of their funding applications, as there is still some lack of clarity on how to approach this.

Patients and members of the public can be anyone who uses or has used healthcare services. This might include parents, informal/formal carers, patient support groups, patient organisations, the general public, charities that represent specific conditions, or individuals with a special interest in the topic that is being researched.

PPI in research means getting any of these people actively involved in the research process itself, rather than just being participants or subjects of the research, with the aim that research is done with or by the public, and not to, about or for them. Thus, PPI does not refer to researchers raising awareness of research, sharing knowledge or recruiting patients as participants, it is more about engaging the members of the public and creating a dialogue with them to drive research forward and make it more patient centred. Although $\mathrm{PPI}$, engagement and participation are different activities, they are often linked and complement each other.

This article aims to give young researchers a basic understanding of PPI in research, its benefits, tips about how to do it, and some "dos and don'ts".

\section{Why should you get patients and members of the public involved?}

Throughout the various phases of clinical research, PPI can potentially improve outcomes, by refining the design and conduct of studies and increasing the dissemination of results. Especially in healthcare services research, given the impact that its outcomes may have in healthcare systems, this may lead to better informed decisions. In the particular case of clinical trials, the goal should be "experimenting with" rather than "experimenting on" patients.

Defining research questions is difficult. It may sometimes be better to work together with patients, understanding and exploring their needs, and converging on a common purpose. PPI in research applies in all studies. However, in specific studies that do not involve individual
Cite as: Hardavella G, Bjerg A, Saad N, et al. Doing science: How to optimise patient and public involvement in your research. Breathe 2015; 11: 223-227 
informed consents from the participants, PPI may become particularly important to ensure openness, transparency and public accountability, through a thorough explanation of the methods used and the results obtained, as well as the impact on their health and/or treatments. PPI may even become an ethical imperative in clinical research, because it supports the concept of "partnership" between patients and researchers, even though this could entail a much more detailed discussion. Table 1 summarises the benefits of PPI in research.

\section{When do they get involved?}

PPI in research can be developed in a variety of different ways throughout the whole research process. Regardless of the stage and level of involvement, it needs to stay active, inclusive and well coordinated. It is worth considering in advance the purposes and circumstances of the involvement, e.g. whether you wish to initiate PPI to help with specific issues or problems arising during the course of a trial, or you wish to initiate it earlier in the research cycle, or are even considering including a patient as a co-investigator. Overall, experience has shown that the earlier PPI is initiated, the better the research outcomes will be.

In detail, patients and members of public can be engaged in:

- Identifying and prioritising the research questions

- Trial development and design, e.g. modifying entry criteria, patient information sheets, ethics applications

- Trial management, e.g. as members of the trial steering committee

- Analysis and interpretation, e.g. feedback about how the relevance of research can benefit future patients

Table 1 Wider benefits of PPI in research

- Identification and clarification of research questions

- Ensuring research questions meet patients' needs

- Improving the design, conduct and dissemination of research studies

- Ensuring openness, transparency and public accountability

- Results in research outcomes that may be better translatable into patient benefit

- Improves patient information, resulting in more appropriate and accessible participant information for would-be participants (language/content)

- Increasing public confidence in health research

- Boosting recruitment

- Enhancing learning from experience and good practice

- Offering different perspectives to the other team members

- Dissemination to wider patient and public audiences
- Dissemination and communication of research findings, e.g. through patient organisations, charities, social media, etc.

Some research groups tend to initiate PPI only for the funding application process, as there are now quite a few funding bodies that rely heavily on evidence of PPI to fund a project. Even though this may seem to be a one-off "tick the box" approach, a project stands better chances of being funded by demonstrating consistent and inclusive PPI throughout the whole project rather than on an ad hoc basis.

It would be fair to ask whether engagement with PPI raises any ethical concerns for the people who are actively participating in the trial PPI. They are actually acting as specialist advisers, providing valuable knowledge based on their experience of a health condition or public health concern; therefore, ethical approval is not needed for the active involvement in the research, e.g. helping to develop a protocol, questionnaire or information sheet, or for being a member of an advisory group, or co-applicant. However, remember that it would not be appropriate to also involve a PPI member in the study as a participant.

\section{How do you find them? How do you select them? How do you organise meetings?}

Finding patients is challenging for every researcher, especially if they are lab based. However, finding the right patients and members of the public for PPI can be even more challenging. First, what matters most is thinking very clearly what level of involvement is required and then which patients or members of the public would be best placed to deliver that. Are you looking for cancer patients? Would you like carers to be involved? Should you invite people from patients' organisations? Generally speaking, the easiest way of getting patients involved is through your own clinics. This group of people is the most straightforward to approach, especially if the patient has been known to you for years and a strong relationship has been built. Additionally, each Trust has a local Research and Design Office and most of them may also have local PPI Leads that can be very helpful in providing links with patients/public members. Clinical Research Networks can also provide valuable input in approaching patients and public members (each country has equivalent offices and authorities with different names).

Alternatively, you can identify patients through flyers and adverts, digital or social media, wordof-mouth through patients and direct contact with patients' organisations. In the European Union, a key stakeholder is the European Lung Foundation (ELF), an organisation dedicated to bringing patients and the public closer to the development 
of respiratory research and guidelines at the European level.

In order to reach these aims, ELF is involved in many different activities within the European Respiratory Society (ERS), including ERS Task Forces, ERS Congress and ERS courses, and in initiatives such as the ELF Patient Priorities Pilot Project (more information is available at www.europeanlung.org).

All junior members who are interested in working with patients on ERS projects should ensure that they discuss the opportunity with ELF and also complete the appropriate section on the application form.

In order to help prepare patients for being involved with professionals, ELF has developed the European Patient Ambassador Programme (EPAP; www.EPAPonline.eu), a free online self-learning programme for patients and carers to gain knowledge and confidence to get involved in different areas of patient involvement. ELF also has a factsheet that could be shared with potential PPI members (available at www.europeanlung.org/en/lungdisease-and-information/factsheets/english) and some healthcare professionals have also found it a valuable tool for their own understanding of PPI. If junior members have opportunities for patients to get involved, they will also be able to use this platform and should contact Kerstin Morrison (Kerstin.Morrison@europeanlung.org) with any enquiries.

Similar initiatives exist in individual countries on both regional and national levels, and many professional national respiratory organisations will have experience in PPI or could help you along the way. In the UK, a valuable source of information is INVOLVE, which is one of the few government-funded programmes of its kind in the world and promotes PPI in the National Health Service, public health and social care research (www.invo.org.uk).

In this year's ERS International Congress in Amsterdam (September 26-30, 2015), participants will have several opportunities to meet with patient organisation groups and network with patients and public members with the potential to initiate future collaborations. Table 2 offers a thorough list of opportunities and dates. Patients will also be taking part in symposia and will be acting as demonstrators in educational courses.

After identifying the potential sources of PPI, you need to specify what level of PPI you seek and who would be the best people to deliver it. Would you like them to be involved in the planning of the research, or solely recruiting? Should you include them in steering committees or establish a patient advisory committee? Commonly, patients and the public have been involved in steering committees for providing feedback on research studies. However, recently patients have been more involved in the design, conduct and recruitment of other participants for research studies. For example, a special website (http://patientsactiveinresearch.org. uk) has been launched by researchers at the University of Oxford in collaboration with local hospitals to bring patients and researchers together to further future research. Based on the level of involvement, you can create a brief "person specification" document and also offer a clear description of what the task would be so as to give a clear idea to people about what they are signing up for. It is also important to clarify whether patients/ public members will require any particular training in order to deliver their "tasks" and whether this is for free or whether there is provision to cover expenses.

Finally, how do you organise meetings? Think what you would like to get out of a meeting and make sure you circulate an agenda at least 1 week before the meeting so that everyone can get prepared and come with ideas/suggestions. The level of PPI will determine the anticipated outcomes of the meeting and these can be variable. In any case you should give clear instructions in lay language,

Table 2 Opportunities during this year's ERS Congress to meet and discuss with patients and members of the public about ongoing PPI in research and future collaborations

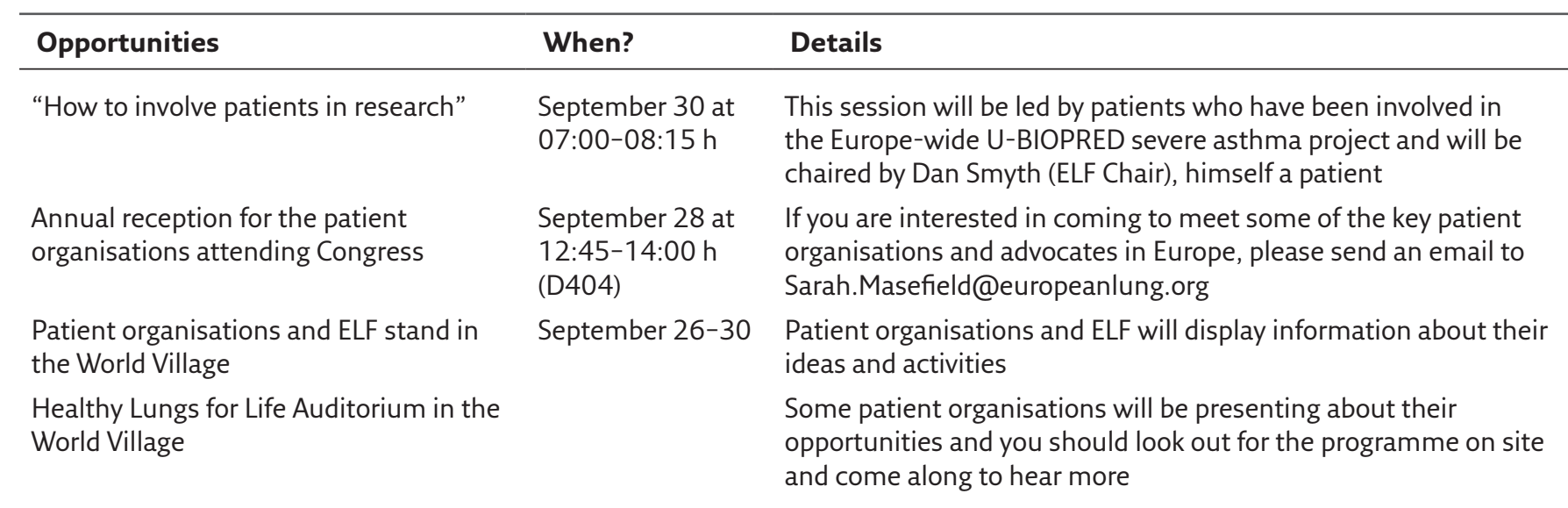

Note that the patient organisation programme is also available on the ERS Congress website (www.erscongress.org). 
especially during the first meeting. Other people taking part in the meeting should also be informed in advance that patients are involved, in order for expectations to be managed. Consider the option of training, should that be required, e.g. in more specialist techniques, study design or medical science for recruitment officers, etc. Consider offering them reimbursement of their travel expenses as a minimum and, if the budget permits, offer reimbursement for their time off work to attend the meeting. However, if funds for your research are tight, consider refunding travel expenses and organise an annual PPI day to thank all the people who helped you during that year, and of course do not forget to send lovely Christmas and Easter cards when the time comes!

\section{Tips for communicating with patients and members of the public: dos and don'ts}

Thinking carefully about what and how you want to communicate with lay people is essential. Not only do you want them to understand the research ideas in order to give their best input, your communication also affects the level of engagement they feel. This "meta-communication" may in fact be equally important. Consider the five following points, which are of importance both at the initial "call" to involve patients and public in your research, and at later stages:

- Openness: Are you really communicating enough high-quality information? Remember that the quantity of information may be sufficient (see "Scope" below), but quality may still be lacking. What would lay people want to know about your research ideas in order to get involved and give their input? Consider involving lay people early, e.g. in the process of creating the initial information material.

- Bias: Is the information you provide neutral? You may be tempted to give limited or selected information in order to get the responses you want. This may also be done subconsciously. Make sure you understand the needs of the people you want to involve and be honest with them.

- Scope: Information overload, even with the best of intentions, is not good for anyone. You need to be direct and to the point. Remember

Table 3 Difficulties and barriers to PPI

- Creating a gap between PPI group and researchers

- Using scientific/very technical language

- Personal difficulties (physical/emotional)

- Language barriers

- Organisational barriers (timing and place of meetings)

- Living with illness and caring responsibilities the last time you signed the dotted line at the end of a 12-page agreement? Or installed new computer software and were faced with tons of fine-print information? Or heard the "sideeffects include" at the end of a pharmaceutical commercial? Don't make the same mistake.

- Flexibility: Which parts of the information you provide are presented as mere facts, and what areas are open for discussion? If this difference is not clear you may encounter trouble down the road. People don't want to spend time and effort on giving constructive criticism to parts of the project you are not willing to change.

- Responsiveness and dialogue: Make sure that patients and the public get polite and adequate replies to their input. You will need to communicate that you have understood the points raised, your view on them and the action(s) taken, as this will build a strong relationship and shows their input is valued. On a similar note, remember to provide regular updates on research progress. Long term, you will find that while some issues are best managed in a closed dialogue with only the lay partner in question, others work better in a plenary format where third parties can engage in the discussion. Consider, for example, the differences between an e-mail conversation and a web forum. Showing others how you manage input from patients and the public can be an excellent way to get more people involved.

Common pitfalls in PPI in research are summarised in table 3. However, they can be reiterated into a tale of two towers that you want to stay clear of:

1) The Tower of Babel. This tower was eventually built so high that its inhabitants no longer spoke the same language. Medicine, just like other highly specialised areas within academia, is laden with in-group jargon and paradigms. These facilitate understanding and communication within the scientific community, but are often obstacles to people outside of it. In addition, they may create or fortify the impression of academic elitism.

2) The Ivory Tower. One big reason why you want to involve lay people is to tackle this home blindness, i.e. that critical input from outside the academic environment may challenge views you took for granted.

\section{Evaluating PPI}

After the dust has settled, one is always coming up with the question "How did it go?" Evaluating PPI is an important activity as it offers information as to whether your original PPI aims and objectives were achieved. However, this is a reciprocal process and, as such, it is also important for the patients and public members that have been involved, as it offers them the opportunity to understand more about the 
impact of their contribution on the research project and how that has been reflected in their own development and future practice. PPI evaluation can also identify what went particularly well (or not) and what could have been done differently, recognise achievements and celebrate success, generate evidence for PPI in research and trigger shared learning that is helpful in designing future projects.

\section{Conflict of interest}

P. Powell is an employee of the European Respiratory Society (ERS).

\section{Suggested reading}

1. Thornton $\mathrm{H}$. Patient and public involvement in clinical trials. BMJ 2008; 336: 903-904.

2. Lilford RJ, Edwards SJL, Braunholtz DA, et al. Ethical issues in the design and conduct of randomised controlled trials. In: Stevens A, Abrams KR, Brazier J, et al., eds. The Advanced Handbook of Methods in Evidence Based Healthcare. London, SAGE Publications Ltd, 2001; pp. 11-24.
3. European Lung Foundation. www.europeanlung.org

4. National Institute for Health Research. INVOLVE. www.invo. org.uk

5. National Institute for Health Research. Patients Active in Research. http://patientsactiveinresearch.org.uk 